\title{
The Dual of a Non-reflexive L-embedded Banach Space Contains $l^{\infty}$ Isometrically
}

by

\author{
Hermann PFITZNER \\ Presented by Stanisław KWAPIEN
}

Summary. A Banach space is said to be L-embedded if it is complemented in its bidual in such a way that the norm between the two complementary subspaces is additive. We prove that the dual of a non-reflexive L-embedded Banach space contains $l^{\infty}$ isometrically.

This note is an afterthought to a result of Dowling [2] according to which a dual Banach space contains an isometric copy of $c_{0}$ if it contains an asymptotic one. (For definitions see below.) It is known ([7] or [4, Th. IV.2.7]) that the dual of a non-reflexive L-embedded Banach space contains $c_{0}$ isomorphically. For a special class of L-embedded Banach spaces the construction of the $c_{0}$-copy has been improved so as to yield an asymptotic one ([8, Prop. 6]) and it turns out that this improvement is possible in the general case, which together with Dowling's result yields isometric copies of $c_{0}$ in the dual of an L-embedded Banach space. As in [7], we will prove a bit more by constructing the $c_{0}$-copy within the context of Pełczyński's property $\left(\mathrm{V}^{*}\right)$, that is, the $c_{0}$-basis will be constructed so as to behave approximately like biorthogonal functionals on the basis of a given $l^{1}$-basis in $X$; see $(3)$ and $(4)$ below where in particular the value $\tilde{c}_{J}\left(x_{n}\right)$ in (3) is optimal. (For the definition and some basic results on Pełczyński's property $\left(\mathrm{V}^{*}\right)$ see [4.)

Preliminaries. A projection $P$ on a Banach space $Z$ is called an $L$ projection if $\|P z\|+\|z-P z\|=\|z\|$ for all $z \in Z$. A Banach space $X$ is called L-embedded (or an L-summand in its bidual) if it is the image of an

2010 Mathematics Subject Classification: Primary 46B20; Secondary 46B03, 46B04, 46B26. Key words and phrases: L-embedded Banach spaces, L-summand, isometric copies of $c_{0}$. 
L-projection on its bidual. In this case we write $X^{* *}=X \oplus_{1} X_{\mathrm{s}}$. Among classical Banach spaces, the Hardy space $H_{0}^{1}, L^{1}$-spaces and, more generally, the preduals of von Neumann algebras or of $\mathrm{JBW}^{*}$-triples serve as examples of L-embedded spaces. A sequence $\left(x_{n}\right)$ in a Banach space $X$ is said to span $c_{0}$ asymptotically isometrically (or just to span $c_{0}$ asymptotically) if there is a null sequence $\left(\delta_{n}\right)$ in $[0,1[$ such that

$$
\sup \left(1-\delta_{n}\right)\left|\alpha_{n}\right| \leq\left\|\sum \alpha_{n} x_{n}\right\| \leq \sup \left(1+\delta_{n}\right)\left|\alpha_{n}\right|
$$

for all $\left(\alpha_{n}\right) \in c_{0} . X$ is said to contain $c_{0}$ asymptotically if it contains such a sequence $\left(x_{n}\right)$. Recall the routine fact that if $\left(x_{n}^{*}\right)$ in $X^{*}$ is equivalent to the canonical basis of $c_{0}$ then $\sum \alpha_{n} x_{n}^{*}$ makes sense for all $\left(\alpha_{n}\right) \in l^{\infty}$ in the $w^{*}$-topology of $X^{*}$, and by lower $w^{*}$-semicontinuity of the norm an estimate $\left\|\sum \alpha_{n} x_{n}^{*}\right\| \leq M \sup \left|\alpha_{n}\right|$ that holds for all $\left(\alpha_{n}\right) \in c_{0}$ extends to all $\left(\alpha_{n}\right) \in l^{\infty}$. The Banach spaces we consider in this note are real or complex; the set $\mathbb{N}$ starts at 1 .

To a bounded sequence $\left(x_{n}\right)$ in a Banach space $X$ we associate its James constant

$$
c_{J}\left(x_{n}\right)=\sup c_{m} \quad \text { where } \quad c_{m}=\inf _{\sum_{n \geq m}\left|\alpha_{n}\right|=1}\left\|\sum_{n \geq m} \alpha_{n} x_{n}\right\|
$$

(the sequence $\left(c_{m}\right)$ is increasing). If $\left(x_{n}\right)$ is equivalent to the canonical basis of $l^{1}$ then $c_{J}\left(x_{n}\right)>0$; more specifically, $c_{J}\left(x_{n}\right)>0$ if and only if there is an integer $m$ such that $\left(x_{n}\right)_{n \geq m}$ is equivalent to the canonical basis of $l^{1}$. (Roughly speaking, the number $c_{J}\left(x_{n}\right)$ may be thought of as the "approximately best $l^{1}$-basis constant" of $\left(x_{n}\right)$; more precisely, there is a null sequence $\left(\tau_{m}\right)$ in $\left[0,1\left[\right.\right.$ (determined by $\left.c_{m}=\left(1-\tau_{m}\right) c_{J}\left(x_{n}\right)\right)$ such that $\left\|\sum_{n=m}^{\infty} \alpha_{n} x_{n}\right\| \geq\left(1-\tau_{m}\right) c_{J}\left(x_{n}\right) \sum_{n=m}^{\infty}\left|\alpha_{n}\right|$ for all $\left(\alpha_{n}\right) \in l^{1}$ and $m \in \mathbb{N}$, and $c_{J}\left(x_{n}\right)$ cannot be replaced by a strictly greater constant.) It is immediate from the definition of the James constant of an $l^{1}$-sequence $\left(x_{n}\right)$ that there are pairwise disjoint finite sets $A_{l} \subset \mathbb{N}$ and a sequence $\left(\lambda_{n}\right)$ of scalars such that $\sum_{k \in A_{l}}\left|\lambda_{k}\right|=1$ and $\tilde{z}_{l} \rightarrow c_{J}\left(x_{n}\right)$ where $\tilde{z}_{l}=\sum_{k \in A_{l}} \lambda_{k} x_{k}$. James' $l^{1}$-distortion theorem states that an appropriate subsequence of the sequence $\left(z_{l}\right)$ defined by $z_{l}=\tilde{z}_{l} /\left\|\tilde{z}_{l}\right\|$ spans $l^{1}$ almost isometrically in the sense that

$$
\left(1-2^{-m}\right) \sum_{l=m}^{\infty}\left|\alpha_{l}\right| \leq\left\|\sum_{l=m}^{\infty} \alpha_{l} z_{l}\right\| \leq\left(1+2^{-m}\right) \sum_{l=m}^{\infty}\left|\alpha_{l}\right|
$$

for all $m \in \mathbb{N}$ and all $\left(\alpha_{n}\right) \in l^{1}$. We will need the fact that if $\left(z_{l}\right)$ spans $l^{1}$ almost isometrically in an L-embedded space $X$ and if $x^{* *} \in X^{* *}$ is a $w^{*}$-accumulation point of the $z_{l}$ then $x^{* *} \in X_{\mathrm{s}}$ and $\left\|x^{* *}\right\|=1$. This follows from the proof of [8, Lem. 1] (or from a more elementary argument proving that $\operatorname{dist}\left(x^{* *}, X\right)=\left\|x_{\mathrm{s}}\right\|=1$ where $\left.x^{* *}=x+x_{\mathrm{s}}\right)$. 
If one passes to a subsequence $\left(x_{n_{k}}\right)$ of $\left(x_{n}\right)$ then $c_{J}\left(x_{n_{k}}\right) \geq c_{J}\left(x_{n}\right)$; hence it makes sense to define

$$
\tilde{c}_{J}\left(x_{n}\right)=\sup _{n_{k}} c_{J}\left(x_{n_{k}}\right) .
$$

The standard reference for L-embedded Banach spaces is the monograph 4, Chap. IV]. For general Banach space theory and undefined notation we refer to [1], [5], or [6].

The main result of this note is

Theorem 1. Let $X$ be an L-embedded Banach space and let $\left(x_{n}\right)$ be equivalent to the canonical basis of $l^{1}$. Then there is a sequence $\left(x_{n}^{*}\right)$ in $X^{*}$ that generates $l^{\infty}$ isometrically, more precisely

$$
\left\|\sum \alpha_{n} x_{n}^{*}\right\|=\sup \left|\alpha_{n}\right| \quad \text { for all }\left(\alpha_{n}\right) \in l^{\infty},
$$

and there is a strictly increasing sequence $\left(p_{n}\right)$ in $\mathbb{N}$ such that

$$
\begin{aligned}
\lim \left|x_{n}^{*}\left(x_{p_{n}}\right)\right| & =\tilde{c}_{J}\left(x_{m}\right), \\
x_{n}^{*}\left(x_{p_{l}}\right) & =0 \quad \text { if } l<n .
\end{aligned}
$$

In particular, the dual of a non-reflexive L-embedded Banach space contains an isometric copy of $l^{\infty}$.

In order to prove the theorem we first state and prove Dowling's result in a way which fits our purpose.

Proposition 2. Let $\left(\varepsilon_{n}\right)$ be a null sequence in $\left[0,1\left[\right.\right.$, let $\left(N_{n}\right)$ be a sequence of pairwise disjoint infinite subsets of $\mathbb{N}$ and let $\left(y_{n}^{*}\right)$ in the dual of a Banach space $Y$ span $c_{0}$ such that

$$
\left\|\sum \alpha_{n} y_{n}^{*}\right\| \leq \sup \left(1+\varepsilon_{n}\right)\left|\alpha_{n}\right| \quad \text { and } \quad\left\|y_{n}^{*}\right\| \rightarrow 1
$$

for all $\left(\alpha_{n}\right) \in c_{0}$. Then the elements

$$
x_{n}^{*}=\sum_{k \in N_{n}} \frac{y_{k}^{*}}{1+\varepsilon_{k}}
$$

generate $l^{\infty}$ isometrically (as in (2)).

Proof. Clearly, $\left\|x_{n}^{*}\right\| \leq 1$ for all $n \in \mathbb{N}$ by the first half of (5). For the inverse inequality we have

$$
\left\|x_{n}^{*}\right\| \geq\left\|2 \frac{y_{m}^{*}}{1+\varepsilon_{m}}\right\|-\left\|\frac{y_{m}^{*}}{1+\varepsilon_{m}}-\sum_{k \in N_{n}, k \neq m} \frac{y_{k}^{*}}{1+\varepsilon_{k}}\right\| \geq 2 \frac{\left\|y_{m}^{*}\right\|}{1+\varepsilon_{m}}-1
$$

for all $m \in N_{n}$, hence $\left\|x_{n}^{*}\right\| \geq 1$ by the second half of (5), which proves $\left\|x_{n}^{*}\right\|=1$. 
Similarly we show (2): First, " $\leq$ " of (2) follows from the first half of (5); second, by the inequality just shown we have

$$
\left\|\sum \alpha_{n} x_{n}^{*}\right\| \geq 2\left|\alpha_{m}\right|-\left\|\alpha_{m} x_{m}^{*}-\sum_{n \neq m} \alpha_{n} x_{n}^{*}\right\| \geq 2\left|\alpha_{m}\right|-\sup \left|\alpha_{n}\right|
$$

for all $m \in \mathbb{N}$, giving " $\geq$ " of (2).

Proof of the Theorem. Let $\left(\delta_{n}\right)$ be a sequence in ]0,1[ converging to 0 . Suppose $\left(x_{n}\right)$ is an $l^{1}$-basis and write $\tilde{c}=\tilde{c}_{J}\left(x_{n}\right)$ for short.

Observation. Given $\tau>0$ there is a subsequence $\left(x_{n_{k}}\right)$ of $\left(x_{n}\right)$ such that $\left|\tilde{c}-c_{J}\left(x_{n_{k}}\right)\right|<\tau$. By James' $l^{1}$-distortion theorem, as described above, there are pairwise disjoint finite sets $A_{l} \subset\left\{n_{k} \mid k \in \mathbb{N}\right\}$ and a sequence $\left(\lambda_{n}\right)$ of scalars such that (1) holds with $\lambda_{n}, \tilde{z}_{l}, z_{l}$ as above; furthermore $\left\|\tilde{z}_{l}\right\| \rightarrow c_{J}\left(x_{n_{k}}\right)$, whence the existence of $l^{\prime}$ such that $\left|\tilde{c}-\left\|\tilde{z}_{l^{\prime}}\right\|\right|<\tau$.

By induction over $n \in \mathbb{N}$ we will construct finite sequences $\left(y_{i}^{(n) *}\right)_{i=1}^{n}$ in $X^{*}$, a sequence $\left(\tilde{y}_{n}\right)$ in $X$, pairwise disjoint finite sets $C_{n} \subset \mathbb{N}$ and a scalar sequence $\left(\mu_{n}\right)$ such that, with the notation $y_{n}=\tilde{y}_{n} /\left\|\tilde{y}_{n}\right\|$,

$$
\sum_{k \in C_{n}}\left|\mu_{k}\right|=1, \quad \tilde{y}_{n}=\sum_{k \in C_{n}} \mu_{k} x_{k}, \quad\left|\tilde{c}-\left\|\tilde{y}_{n}\right\|\right|<\delta_{n},
$$

$$
\left|y_{i}^{(n) *}\left(y_{i}\right)\right|>1-\delta_{i} \quad \forall i \leq n,
$$

$$
y_{i}^{(n) *}\left(y_{l}\right)=0 \quad \forall l<i \leq n,
$$

$$
y_{i}^{(n) *}\left(x_{p}\right)=0 \quad \forall p \in C_{l}, \forall l<i \leq n,
$$

$$
\left\|\sum_{i=1}^{m} \alpha_{i} y_{i}^{(n) *}\right\| \leq \max _{i \leq m}\left(1+\left(1-2^{-n}\right) \delta_{i}\right)\left|\alpha_{i}\right| \quad \forall m \leq n, \alpha_{i} \text { scalars. }
$$

For $n=1$ we use the observation above with $\tau=\delta_{1}$ and choose $l_{1}$ such that ||$\left|\tilde{z}_{l_{1}} \|-\tilde{c}\right|<\delta_{1}$. Then we choose $y_{1}^{(1) *}$ such that $\left\|y_{1}^{(1) *}\right\|=1$ and $y_{1}^{(1) *}\left(z_{l_{1}}\right)$ $=\left\|z_{l_{1}}\right\|$. It remains to set $C_{1}=A_{l_{1}}, \mu_{k}=\lambda_{k}$ for $k \in C_{1}$ and $\tilde{y}_{1}=\tilde{z}_{l_{1}}$.

For the induction step $n \mapsto n+1$ we recall that $\left(P^{*}\right)_{\mid X^{*}}$ is an isometric isomorphism from $X^{*}$ onto $X_{\mathrm{s}}^{\perp}$, that $X^{* * *}=X^{\perp} \oplus_{\infty} X_{\mathrm{s}}^{\perp}$ and that $\left(P^{*} x^{*}\right)_{\mid X}=\left(x^{*}\right)_{\mid X}$ for all $x^{*} \in X^{*}$. Let $\left(z_{l}\right)$ be as in the observation above with $\tau=\delta_{n+1}$ and let $z_{\mathrm{s}} \in X^{* *}$ be a $w^{*}$-accumulation point of the $z_{l}$. Then $z_{\mathrm{s}} \in X_{\mathrm{s}}$ and $\left\|z_{\mathrm{s}}\right\|=1$ (as explained in the preliminaries). Choose $t \in \operatorname{ker} P^{*} \subset X^{* * *}$ such that $\|t\|=1$ and $t\left(z_{\mathrm{s}}\right)=\left\|z_{\mathrm{s}}\right\|$. Put

$$
\begin{aligned}
& E=\operatorname{lin}\left(\left\{P^{*} y_{i}^{(m) *} \mid i \leq m \leq n\right\} \cup\{t\}\right) \subset X^{* * *}, \\
& F=\operatorname{lin}\left(\left\{z_{\mathrm{s}}\right\} \cup\left\{x_{p} \mid p \in \bigcup_{l \leq n} C_{l}\right\}\right) \subset X^{* *}
\end{aligned}
$$

and choose $\eta>0$ such that

$$
(1+\eta)\left(1+\left(1-2^{-n}\right) \delta_{i}\right)<1+\left(1-2^{-(n+1)}\right) \delta_{i} \quad \text { and } \quad \eta<\left(1-2^{-(n+1)}\right) \delta_{n+1}
$$


for all $i \leq n$. The principle of local reflexivity provides an operator $R: E \rightarrow$ $X^{*}$ such that

$$
\begin{aligned}
(1-\eta)\left\|e^{* * *}\right\| & \leq\left\|R e^{* * *}\right\| \leq(1+\eta)\left\|e^{* * *}\right\|, \\
f^{* *}\left(R e^{* * *}\right) & =e^{* * *}\left(f^{* *}\right),
\end{aligned}
$$

for all $e^{* * *} \in E$ and $f^{* *} \in F$.

We define $y_{i}^{(n+1) *}=R\left(P^{*} y_{i}^{(n) *}\right)$ for $i \leq n$ and $y_{n+1}^{(n+1) *}=R t$ and obtain (11) $n+1$ (with $\alpha_{i}=0$ if $\left.m<i \leq n+1\right)$ by

$$
\begin{aligned}
\left\|\sum_{i=1}^{n+1} \alpha_{i} y_{i}^{(n+1) *}\right\| & \stackrel{\sqrt[12]{12}}{\leq}(1+\eta)\left\|\left(\sum_{i=1}^{n} \alpha_{i} P^{*} y_{i}^{(n) *}\right)+\alpha_{n+1} t\right\| \\
& =(1+\eta) \max \left(\left\|\sum_{i=1}^{n} \alpha_{i} P^{*} y_{i}^{(n) *}\right\|,\left\|\alpha_{n+1} t\right\|\right) \\
& =(1+\eta) \max \left(\left\|\sum_{i=1}^{n} \alpha_{i} y_{i}^{(n) *}\right\|,\left\|\alpha_{n+1} t\right\|\right) \\
& \leq(1+\eta) \max \left(\max _{i \leq n}\left(1+\left(1-2^{-n}\right) \delta_{i}\right)\left|\alpha_{i}\right|,\left|\alpha_{n+1}\right|\right) \\
& \leq \max _{i \leq n+1}\left(1+\left(1-2^{-(n+1)}\right) \delta_{i}\right)\left|\alpha_{i}\right| .
\end{aligned}
$$

Since $z_{\mathrm{s}}$ is a $w^{*}$-cluster point of $\left(z_{l}\right)$ we have

$$
\begin{aligned}
\left|y_{n+1}^{(n+1) *}\left(z_{l}\right)\right| & >\left|z_{\mathrm{s}}\left(y_{n+1}^{(n+1) *}\right)\right|-\delta_{n+1} \\
& \stackrel{130}{=}\left|t\left(z_{\mathrm{s}}\right)\right|-\delta_{n+1}=1-\delta_{n+1}
\end{aligned}
$$

for infinitely many $l$; furthermore, an $l_{n+1}$ can be chosen among those $l$ so as to obtain ||$\left|\tilde{z}_{l_{n+1}} \|-\tilde{c}\right|<\delta_{n+1}$. Set $C_{n+1}=A_{l_{n+1}}, \tilde{y}_{n+1}=\tilde{z}_{l_{n+1}}, \mu_{k}=\lambda_{k}$ for $k \in C_{n+1}$. Then $(7)_{n+1}$ holds and $(8)_{n+1}$ holds for $i=n+1$. For $i \leq n$, (8) $n+1$ follows from

$$
y_{i}^{(n+1) *}\left(y_{i}\right)=\left(P^{*} y_{i}^{(n) *}\right)\left(y_{i}\right)=y_{i}^{(n) *}\left(y_{i}\right) \stackrel{8}{>} 1-\delta_{i} .
$$

Condition $10 p_{n+1}$ holds for $i=n+1$ by

$$
y_{n+1}^{(n+1) *}\left(x_{p}\right)=(R t)\left(x_{p}\right) \stackrel{13}{=} t\left(x_{p}\right)=0 \quad \forall p \in C_{l}, \forall l<n+1
$$

and it holds for $i<n+1$ by

$$
y_{i}^{(n+1) *}\left(x_{p}\right)=\left(P^{*} y_{i}^{(n) *}\right)\left(x_{p}\right)=y_{i}^{(n) *}\left(x_{p}\right) \stackrel{100}{=} 0 \quad \forall p \in C_{l}, \forall l<i .
$$

Condition $(9)_{n+1}$ follows from $10 n_{n+1}$. This ends the induction.

Now we define $y_{i}^{*}=\frac{1}{1+\delta_{i}} \lim _{n \in \mathcal{U}} y_{i}^{(n) *}$ for all $i \in \mathbb{N}$ where $\mathcal{U}$ is a fixed nontrivial ultrafilter on $\mathbb{N}$ and where the limit is understood in the $w^{*}$-topo- 
logy of $X^{*}$. Then by $w^{*}$-lower semicontinuity of the norm and by (11),

$$
\left\|\sum \alpha_{i} y_{i}^{*}\right\| \leq \sup \left(1+\delta_{i}\right) \frac{\left|\alpha_{i}\right|}{1+\delta_{i}}=\sup \left|\alpha_{i}\right|
$$

for all $\left(\alpha_{i}\right) \in l^{\infty}$. In particular, $\left\|y_{i}^{*}\right\| \leq 1$, hence $\left\|y_{i}^{*}\right\| \rightarrow 1$ by (8) and $\left(y_{i}^{*}\right)$ satisfies (5) for $\varepsilon_{n}=0$.

Let $\left(N_{n}\right)$ be a sequence of pairwise disjoint infinite subsets of $\mathbb{N}$ such that $\left(i_{n}\right)$ increases strictly where $i_{n}=\min N_{n}$. By the proposition the sequence defined by

$$
x_{n}^{*}=\sum_{i \in N_{n}} y_{i}^{*}
$$

generates $l^{\infty}$ isometrically and we have

$$
\left|x_{n}^{*}\left(y_{i_{n}}\right)\right| \stackrel{\text { 9 }}{=}\left|y_{i_{n}}^{*}\left(y_{i_{n}}\right)\right| \stackrel{8}{\geq} \frac{1-\delta_{i_{n}}}{1+\delta_{i_{n}}} .
$$

By construction of the $y_{i}$ there is, for each $n \in \mathbb{N}$, an index $p_{n} \in C_{i_{n}}$ such that

$$
\left(1+\delta_{i_{n}}\right)\left|x_{n}^{*}\left(x_{p_{n}}\right)\right| \geq\left(1-\delta_{i_{n}}\right)\left\|\tilde{y}_{i_{n}}\right\| \stackrel{(7 p}{\geq}\left(1-\delta_{i_{n}}\right)\left(\tilde{c}-\delta_{i_{n}}\right),
$$

which will yield " $\geq$ " of (3). In order to show " $\leq$ " of (3) suppose to the contrary that $x_{n_{m}}^{*}\left(x_{p_{n_{m}}}\right)>\kappa+\tilde{c}$ for appropriate subsequences, all $m$ and $\kappa>0$. According to an extraction lemma of Simons [10] we may furthermore suppose that $\sum_{j \neq m}\left|x_{n_{j}}^{*}\left(x_{p_{n_{m}}}\right)\right|<\kappa / 2$ for all $m$. Then given $\left(\alpha_{m}\right)$ and $\theta_{m}$ such that $\theta_{m} \alpha_{m}=\left|\alpha_{m}\right|$ we obtain

$$
\begin{aligned}
\left\|\sum \alpha_{m} x_{p_{n_{m}}}\right\| & \geq\left(\sum_{j} \theta_{j} x_{n_{j}}^{*}\right)\left(\sum_{m} \alpha_{m} x_{p_{n_{m}}}\right) \\
& \geq(\kappa+\tilde{c}) \sum_{m}\left|\alpha_{m}\right|-\sum_{m} \sum_{j \neq m}\left|\alpha_{m}\right|\left|x_{n_{j}}^{*}\left(x_{p_{n_{m}}}\right)\right| \\
& \geq(\kappa / 2+\tilde{c}) \sum_{m}\left|\alpha_{m}\right|,
\end{aligned}
$$

which yields the contradiction $c_{J}\left(x_{p_{n_{m}}}\right)>\tilde{c}$ and thus shows " $\leq$ " and all of (3), whereas (4) follows from (10) via $y_{i}^{*}\left(x_{p}\right)=0$ for $p \in C_{l}, l<i$.

The last assertion of the theorem is immediate from the fact that nonreflexive L-embedded spaces contain $l^{1}$ isomorphically [4, IV.2.3].

REMARKs. 1. It is not clear whether (4) can be obtained also for $l>n$. What can be said by Simons' extraction lemma (used in the proof) is that, under the assumptions of the theorem and given $\varepsilon>0$, it is possible (after passing to appropriate subsequences) to deduce in addition to (4) that $\sum_{n=1}^{l-1}\left|x_{n}^{*}\left(x_{p_{l}}\right)\right|=\sum_{n \neq l}\left|x_{n}^{*}\left(x_{p_{l}}\right)\right|<\varepsilon$ for all $l$. In case $\tilde{c}_{J}\left(x_{n}\right)=1=\lim \left\|x_{n}\right\|$ 
(which happens when the $x_{n}$ span $l^{1}$ almost isometrically) this can be improved to

$$
\sum_{n \neq l}\left|x_{n}^{*}\left(x_{p_{l}}\right)\right|=\left(\sum_{n}\left|x_{n}^{*}\left(x_{p_{l}}\right)\right|\right)-\left|x_{l}^{*}\left(x_{p_{l}}\right)\right| \leq\left\|x_{p_{l}}\right\|-\left|x_{l}^{*}\left(x_{p_{l}}\right)\right| \rightarrow 0 .
$$

One might also construct straightforward perturbations of the $x_{n}^{*}$ in order to get (4) for $l \neq n$ but then it is not clear whether these perturbations can be arranged to span $c_{0}$ isometrically, not just almost isometrically.

Since in general L-embedded spaces do not contain $l^{1}$ isometrically (see below, last remark) it is not in general possible, in case all $x_{n}$ have the same norm, to improve (3) and (4) so as to obtain $x_{n}^{*}\left(x_{p_{l}}\right)=\tilde{c}\left(x_{m}\right)$ if $l=n$ and $=0$ if $l \neq n$.

2. As already alluded to in the introduction, the construction of $c_{0}$ in this paper bears much resemblance to the one of [7]. A different way to construct $c_{0}$ is contained in [9] but it seems unlikely that this construction can be improved to yield an isometric $c_{0}$-copy.

3. It follows from (3) (or rather from a reasoning similar to the one in (14)) that $c\left(x_{p_{n}}\right) \geq \tilde{c}\left(x_{n}\right)$, which means that in L-embedded spaces the sup in the definition of $\tilde{c}_{J}$ is attained by the James constant of an appropriate subsequence. For general Banach spaces this is not known, although it can be shown by a routine diagonal argument that each bounded sequence $\left(x_{n}\right)$ admits a $c_{J}$-stable subsequence $\left(x_{n_{k}}\right)$ (meaning that $\tilde{c}_{J}\left(x_{n_{k}}\right)=c_{J}\left(x_{n_{k}}\right)$ ) whose James constant is arbitrarily near to $\tilde{c}\left(x_{n}\right)$.

4. Each normalized sequence $\left(x_{n}\right)$ in an L-embedded Banach space that spans $l^{1}$ almost isometrically contains a subsequence each of whose $w^{*}$ accumulation points in the bidual attains its norm on the dual unit ball. To see this, let $\left(x_{n}^{*}\right)$ and $\left(x_{p_{n}}\right)$ be the sequences given by the theorem and by Simons' extraction lemma (see (15) above), let $x_{\mathrm{s}}$ be a $w^{*}$-accumulation point of the $x_{p_{n}}$ and let $x^{*}=\sum x_{n}^{*}$; then $\left\|x^{*}\right\|=1$ and on the one hand $\left\|x_{\mathrm{s}}\right\|=1$ by [8] and on the other hand $x_{\mathrm{s}}\left(x^{*}\right)=\lim x^{*}\left(x_{p_{n}}\right) \stackrel{15}{=} \lim x_{n}^{*}\left(x_{p_{n}}\right) \stackrel{\text { (3) }}{=} c_{J}\left(x_{n}\right)=1$.

It would be interesting to know whether this remark holds for the whole sequence $\left(x_{n}\right)$ instead of only a subsequence $\left(x_{p_{n}}\right)$. A kind of converse follows from [9, Rem. 2] for separable $X$ : If $x_{\mathrm{s}} \in X_{\mathrm{s}}$ attains its norm on the dual unit ball then it does so on the sum of a wuC-series.

5 . Let us finally note that the presence of isometric $c_{0}$-copies in $X^{*}$ does not necessarily entail the presence of isometric copies of $l^{1}$ in $X$ even if $X$ is the dual of an M-embedded Banach space. This follows from [4, Cor. III.2.12], which states that there is an L-embedded Banach space which is the dual of an M-embedded space (to wit, the dual of $c_{0}$ with an equivalent norm) which is strictly convex and therefore does not contain $l^{1}$ isometrically although it contains, as do all non-reflexive L-embedded spaces, $l^{1}$ asymptotically ([8], 
see [3] for the definition of asymptotic copies $\left({ }^{1}\right)$ and the difference from almost isometric ones).

\section{References}

[1] J. Diestel, Sequences and Series in Banach Spaces, Springer, Berlin, 1984.

[2] P. N. Dowling, Isometric copies of $c_{0}$ and $\ell^{\infty}$ in duals of Banach spaces, J. Math. Anal. Appl. 244 (2000), 223-227.

[3] P. N. Dowling, W. B. Johnson, C. J. Lennard, and B. Turett, The optimality of James's distortion theorems, Proc. Amer. Math. Soc. 125 (1997), 167-174.

[4] P. Harmand, D. Werner, and W. Werner, $M$-ideals in Banach Spaces and Banach Algebras, Lecture Notes in Math. 1547, Springer, 1993.

[5] W. B. Johnson and J. Lindenstrauss (eds.), Handbook of the Geometry of Banach Spaces, Volumes 1 and 2, North-Holland, 2001, 2003.

[6] J. Lindenstrauss and L. Tzafriri, Classical Banach Spaces I and II, Springer, Berlin, 1977, 1979.

[7] H. Pfitzner, L-summands in their biduals have Pełczyński's property $\left(\mathrm{V}^{*}\right)$, Studia Math. 104 (1993), 91-98.

[8] -, A note on asymptotically isometric copies of $l^{1}$ and $c_{0}$, Proc. Amer. Math. Soc. 129 (2001), 1367-1373.

[9] - Separable L-embedded Banach spaces are unique preduals, Bull. London Math. Soc. 39 (2007), 1039-1044.

[10] S. Simons, On the Dunford-Pettis property and Banach spaces that contain $c_{0}$, Math. Ann. 216 (1975), 225-231.

Hermann Pfitzner

Université d'Orléans

BP 6759

F-45067 Orléans Cedex 2, France

E-mail: hermann.pfitzner@univ-orleans.fr

Received January 8, 2010;

received in final form March 25, 2010

$\left({ }^{1}\right)$ In the literature there is another notion of "asymptotic $l^{p}$ " which is quite different from the one of this note. 